\title{
Low-temperature specific heat and glassy dynamics of a polymorphic molecular solid
}

\author{
C. Talón, M. A. Ramos, and S. Vieira \\ Laboratorio de Bajas Temperaturas, Departamento de Física de la Materia Condensada, C-III, Universidad Autónoma de Madrid, \\ Cantoblanco, E-28049 Madrid, Spain
}

G. J. Cuello, F. J. Bermejo, A. Criado, and M. L. Senent

Consejo Superior de Investigaciones Científicas, Serrano 123, E-28006 Madrid, Spain

S. M. Bennington

Rutherford Appleton Laboratory, Chilton, Didcot, Oxon, OX11, OQX, Great Britain

H. E. Fischer and H. Schober

Institut Laue Langevin, BP 156x, F-38042 Grenoble Cedex 9, France

(Received 14 January 1998)

\begin{abstract}
The low-frequency dynamics of different solid phases of ethanol (fully ordered crystal, orientational glass, and true structural glass) is investigated by measurements of low-temperature specific heat as well as determination of the vibrational densities of states by inelastic neutron scattering. Such polymorphism provides a unique benchmark to study the relative importance of different kinds of disorder on glass-dynamics properties. The measurements are carried out both for hydrogenous and fully-deuterated samples. Large isotopic effects are found for the excess contributions (i.e., non-Debye) to the specific heat of the disordered solids, which have clear correlates in the low-frequency spectra. [S0163-1829(98)05626-4]
\end{abstract}

\section{INTRODUCTION}

From the pioneering work by Zeller and Pohl, ${ }^{1}$ it has been established that the low-temperature thermal properties of glasses show a universal behavior, ${ }^{1-3}$ which significantly differs from that observed in fully ordered crystals. Since then, the term "glassy" has come to describe in many materials any behavior associated with the loss of some long-range spatial regularity, such as anomalies in the elastic properties, ${ }^{4}$ an excess of vibrational modes at low frequencies with respect to the crystal spectrum (known as the boson peak), or the characteristic relaxation behavior ${ }^{5}$ observed near the glass-transition temperature $T_{g}$. In fact, below $1 \mathrm{~K}$, the specific heat $C_{p}$ of glasses is much larger and the thermal conductivity $\kappa$ far lower than those found in their crystalline counterparts, $C_{p}$ depending almost linearly and $\kappa$ almost quadratically on temperature. Above $1 \mathrm{~K}, C_{p}$ still deviates strongly from the expected Debye $T^{3}$ dependence of the specific heat due to acoustic phonons, exhibiting a remarkable broad maximum in a $C_{p} / T^{3}$ vs $T$ plot. In the same temperature range, the thermal conductivity exhibits a ubiquitous plateau. Whereas the region of very low temperatures was soon understood ${ }^{3}$ on the basis of the phenomenological tunneling model, ${ }^{6}$ the latter still constitutes a matter of debate. ${ }^{7}$ Moreover, the distinctive behavior of disordered matter is not confined to lower temperatures, as inspection of the behavior of $\kappa(T \geqslant 50 \mathrm{~K})$ clearly shows. ${ }^{8}$ On the other hand, it is above $1 \mathrm{~K}$ where a connection between the thermal properties and the atomic dynamics as described by the vibrational spectrum of the glass can be established, which provides a very valuable key for the microscopic investigation of the low-energy dynamics of glasses and supercooled liquids. ${ }^{9}$
The other major research topic concerns the very nature of the glass-transition phenomenon, when the supercooled liquid falls from metastable equilibrium into a disordered solid. Many questions remain unanswered, but a consensus has been reached in the scientific community ${ }^{9}$ stating that both the "solid-state," low-temperature anomalies, and the "liquid-state" behavior of the glass around the glasstransition temperature $T_{g}$, should be considered jointly in order to achieve a complete understanding of the glassy state. ${ }^{10,11}$ Although some attempts ${ }^{12,13}$ have been made to relate (at least, phenomenologically) the low-temperature properties of glasses and the boson peak with the glass transition, it is undoubtedly an open question and a current matter of vivid discussions.

The lack of translational order in conventional glasses (i.e., amorphous solids) and the corresponding complexity of their microscopic structure and dynamics, have led to alternative ways of addressing the question, based upon the search for glassy behavior in crystalline solids with some kind of disorder. Following this idea, much experimental and theoretical efforts have been devoted, for instance, to alkali cyanide mixed crystals ${ }^{14-16}$ [i.e., $\left.(\mathrm{KBr})_{1-x}(\mathrm{KCN})_{x}\right]$. These are crystals grown with a controlled amount of orientational disorder, which is governed by competing interactions between the spherical halogen sites and the cyanide ions, leading to an orientationally disordered state at high cyanide concentrations. ${ }^{17}$ The latter have been found to exhibit lowtemperature specific heat and thermal conductivity behaviors akin to those of structurally disordered, amorphous solids. ${ }^{15}$ A class of apparently similar solids is that of glass-forming plastic crystals $^{18,19}$ that exhibit orientational disorder of dynamic origin. An orientational glass (OG) state can be 


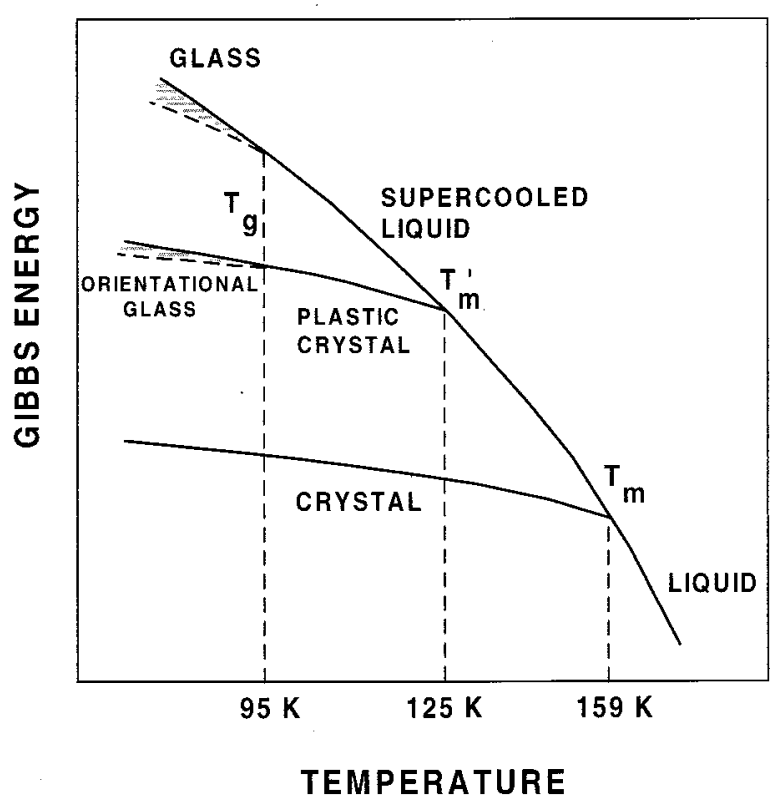

FIG. 1. Schematic phase diagram of ethanol.

reached by supercooling a high-symmetry crystalline phase where molecules are rotating (plastic crystal or rotator phase). These materials exhibit polymorphism, since a lower-symmetry (typically monoclinic) crystalline phase must be bypassed in order to reach that disordered state. In contrast to orientationally-disordered mixed crystals, orientational glasses do show a glass-transition like true structural glasses, and they are stoichiometrically homogeneous materials.

Unfortunately, most substances that can be prepared as orientational glasses cannot be produced easily in an amorphous phase, ${ }^{18,19}$ which hinders any quantitative comparison between the anomalies of both types of disordered phases. Nevertheless, at least one material exists that can be prepared readily in all the relevant phases. As first shown by Haida and co-workers, ${ }^{20}$ ethanol $\left(\mathrm{CH}_{3} \mathrm{CH}_{2} \mathrm{OH}\right)$ exhibits a rich polymorphism, since it can be obtained either as a stable crystal, as an ordinary glass, or as a disordered crystal with glassy properties, depending upon temperatures and cooling rates below its melting point at $T_{m}=159 \mathrm{~K}$. More recent studies $^{21,22}$ have clarified the nature of this polymorphism. In fact, there exist four different solid-state phases at ambient pressure (see Fig. 1): the stable (monoclinic) crystal, a plastic (bcc) crystal with rotational disorder or rotator phase (RP), an orientational glass $(\mathrm{OG})$ obtained by quenching the RP, and finally the true structural glass obtained by fast cooling of the liquid. Interestingly, the "glass transition" corresponding to the freezing of the RP crystal into the OG state takes place at the same temperature $(\approx 95 \mathrm{~K})$ and the jump in specific heat is of comparable magnitude to that shown at the ordinary liquid-glass transition. ${ }^{20}$ Furthermore, it has been found $^{23,24}$ that the orientational glass phase exhibits lowtemperature properties close to those of the structural glass (a broad maximum in $C_{p} / T^{3}$ below $10 \mathrm{~K}$ and a quasilinear contribution at the lowest temperatures, a boson peak in the vibrational spectrum, etc.) On the contrary, the stable crystal shows the expected Debye behavior at low temperatures and energies.
Ethanol thus constitutes a unique benchmark sample to assess the roles of positional and orientational disorder in the low-energy dynamics and hence in the low-temperature properties of glasses or amorphous solids. Moreover, it should help to gain insight into the microscopic nature of those low-energy excitations, since one can rely first on the existence of an underlying crystalline lattice (in the RP or OG phases) that facilitates the analysis of many physical properties, and then add the positional disorder to that orientationally-disordered material by passing to the glassy state.

An additional point of interest for all those disordered materials where the internal dynamics of the constituent particles cannot be fully decoupled from the collective motions, regards the interplay between "molecular" or fairly localized excitations with others of more extended nature. The question seems of utmost relevance for a fairly large class of glassy materials (complex organics, polymers, etc.) whose low-frequency dynamics have been scrutinized in some detail in recent times.

In this paper we address these questions from the study of the low-temperature specific heat between 1.8 and $30 \mathrm{~K}$, together with calorimetric characterizations at temperatures about the transitions $(80-130 \mathrm{~K})$ performed using the same experimental cell, as well as with the measurement of the spectral distributions [i.e., vibrational densities of states (VDOS's)] recently carried out by means of inelastic neutron-scattering experiments at low temperatures. Having access to the fully-ordered crystal enables us (a) to define an absolute frequency scale to which all relevant motions are referred, (b) to attempt a full characterization of the crystal dynamics by means of the combined use of experiment and lattice dynamics (LD's) calculations, and (c) to compare at least in a semiquantitative way the departure of the dynamics from the fully-ordered behavior introduced by the subsequent loss of orientational and positional regularities. Furthermore, the nature and extent of mode hybridization between internal (intramolecular) and external vibrations is addressed by the investigation of the isotopic effect that is enabled by the use of both hydrogenated $\left(\mathrm{CH}_{3} \mathrm{CH}_{2} \mathrm{OH}\right)$ and fully-deuterated $\left(\mathrm{CD}_{3} \mathrm{CD}_{2} \mathrm{OD}\right)$ samples.

\section{EXPERIMENTAL AND COMPUTATIONAL DETAILS}

\section{A. Calorimetric measurements}

In order to achieve the required accuracy, we have built a low-temperature calorimetric cell different from that employed in previous experiments. ${ }^{23,24}$ The setup consists in a quasiadiabatic, vacuum-tight copper sample can, suspended by nylon threads from the inner flange of a double-chamber insert, with an additional vessel to pump helium, operating within a double-walled glass cryostat. A precalibrated germanium thermometer (Cryo Cal, MCR-50) suited for low temperatures, a standard silicon diode for the whole temperature range, and an electrical heater were attached to the thinwalled copper can. To facilitate the rapid attainment of thermal equilibrium inside the can (i.e., the ethanol sample), a thin copper mesh was fitted inside the sample container. A simple mechanical heat switch was used to cool the experimental cell. This consisted in a thin gold wire glued to the 


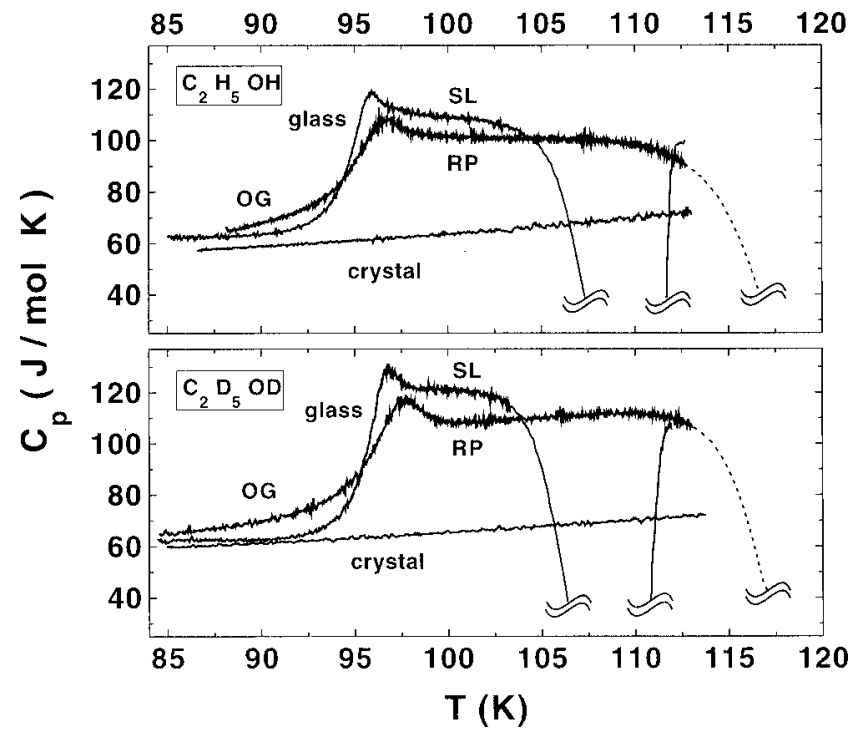

FIG. 2. Specific heat in the glass-transition temperature region for all the ethanol phases studied in this work, measured at heating rates of about $+0.2 \mathrm{~K} / \mathrm{min}$. Upper panel: normal ethanol. Lower panel: fully-deuterated ethanol. OG: orientational glass. RP: rotator phase. SL: supercooled liquid. Dashed lines indicate the irreversible crystallization processes taking place, which are therefore monitored in situ during these experimental runs. The phase so prepared will be the next to be measured at low temperatures and subsequently characterized here by the same procedure (see text for further details.)

copper can. After filling completely the container with ethanol (typically $2 \mathrm{~cm}^{3}$ ), the can is mechanically sealed.

All the specific-heat measurements, including those around $100 \mathrm{~K}$ to characterize the phases, were carried out using the same experimental cell. The latter was carefully weighted before and after being emptied. The heat capacity of the empty cell was then measured in order to subtract the addenda contribution. At $4.2 \mathrm{~K}$, the addenda contributed about $10 \%$ for the glassy states, and about $25 \%$ for the crystals, to the total measured heat capacity. It is worth mentioning that different sets of experiments and also slightly different thermal histories and environments gave indistinguishable experimental results, therefore supporting the alluded accuracy of the measurements presented below. Commercially available pure ethanol $\mathrm{CH}_{3} \mathrm{CH}_{2} \mathrm{OH}$ (Merck, max. $0.02 \% \mathrm{H}_{2} \mathrm{O}$ ) and fully-deuterated ethanol $\mathrm{CD}_{3} \mathrm{CD}_{2} \mathrm{OD}$ (Cambridge Isotope Laboratories, anhydrous $99 \% \mathrm{D}$ ) were used, without further purification. The procedure followed to prepare, measure, and characterize the different solid phases of ethanol can be understood by recourse to Figs. 1 and 2 . After attaining thermal equilibrium for liquid ethanol slightly above its melting point at $T_{m}=159 \mathrm{~K}$, the calorimetric cell containing the ethanol is cooled faster than $-20 \mathrm{~K} / \mathrm{min}$ down to below $95 \mathrm{~K}$, so that any kind of crystallization is avoided and the supercooled liquid falls into the glass state. To achieve high quenching rates, the abovementioned thermal switch was supplemented by a small amount of helium exchange gas that was allowed to enter the inner chamber to enhance thermal contact between the cell and the thermal bath. This residual helium gas is later evacuated completely. To achieve this, the chamber was pumped at liquid-nitrogen temperatures for more than $24 \mathrm{~h}$. Then, liquid helium was used to cool down the experimental system, with only the help of the mechanical heat switch.

After performing the specific-heat measurements on the glass from 1.8 to $30 \mathrm{~K}$, the system is heated up to liquidnitrogen temperatures and the heat capacity is measured using the continuous method at heating rates of about +0.2 $\mathrm{K} / \mathrm{min}$, with the aim of characterizing the phase previously obtained and measured (see Fig. 2). A jump ${ }^{25}$ in the specific heat is observed due to the release of configurational degrees of freedom typical of the glass-transition phenomenon. Taking the standard definition of calorimetric glass-transition temperature $T_{g}$ as the temperature where the experimental curve reaches the midpoint of the specific-heat jump to the liquid-state value well above $T_{g}$, a value of $T_{g}=95 \mathrm{~K}$ is obtained both for normal and deuterated ethanol glasses, in excellent agreement with previous data. ${ }^{20,26}$ By further heating the supercooled liquid, a phase transition into the plastic crystal or RP irreversibly occurs within the $105-110 \mathrm{~K}$ temperature window. The estimated enthalpy of the observed transition is also in good agreement with that reported by Haida and co-workers, ${ }^{20}$ confirming that we had indeed produced $100 \%$ of the glass phase. By further cooling this phase after the transition has been completed, we obtained the OG to be measured at low temperature. Repetition of the previous procedure, ended on a similar "glass transition" at $95 \mathrm{~K}$ with a jump of about $80 \%$ of that of the true liquid-glass transition, again in agreement with previous measurements on this "glassy crystal" of normal ethano ${ }^{20}$ and of fullydeuterated ethanol. ${ }^{26}$ As discussed below, this is strictly speaking a $\mathrm{RP} \rightarrow \mathrm{OG}$ freezing transition, rather than an ordinary glass transition. When further heated, ethanol does not show any trace of a phase transition in the 105-110 K interval, therefore confirming again that we had obtained $100 \%$ of the RP material. When the RP or (bcc) plastic crystal is further heated up to about $120 \mathrm{~K}$, a transition into the (monoclinic) stable crystal phase occurs, which is mediated by the very rapid melting of the RP crystal. This is then an alternative, very convenient method to produce the stable crystal, instead of slowly cooling the liquid below $T_{m}$. Once again, the phase is cooled down to liquid-helium temperatures, the specific heat of crystalline ethanol is measured, and it is subsequently heated to about $85 \mathrm{~K}$, where heat-capacity data (see Fig. 2) do not show any evidence of glassy behavior, as expected. We want to point out that absolute accuracy in "high-temperature" specific-heat measurements of Fig. 2 is not expected to be so good as in low-temperature ones, for which the experimental cell has been designed. Nevertheless, the relative precision and temperature resolution suffice for the present purposes.

\section{B. Neutron scattering}

Two sets of inelastic neutron-scattering (INS) experiments were carried out: one of them on the IN5 spectrometer at the High Flux Reactor of the Institut Laue-Langevin, Grenoble, France, exploring in detail the low-energy range $(\hbar \omega<1 \mathrm{THz})$; and the other on the MARI spectrometer at the ISIS pulsed neutron source at the Rutherford Appleton Laboratory, Chilton Didcot, United Kingdom, exploring the energy range between 0.5 and $15 \mathrm{THz}$. In both of them, the 
samples consisted of $\mathrm{CH}_{3} \mathrm{CH}_{2} \mathrm{OH}$ and $\mathrm{CD}_{3} \mathrm{CD}_{2} \mathrm{OD}$, and the same samples were employed for measurements of the different solid phases.

For the high-resolution experiments on the IN5 spectrometer the samples were enclosed within flat plate aluminum containers that were subsequently mounted into the center stick of an standard orange cryostat. The four phases were prepared at ease in situ as checked by inspection of the diffraction pattern. ${ }^{27}$ Measurements in the different phases were taken at relatively low temperatures $(5$ and $20 \mathrm{~K})$ in order to establish an unambiguous comparison with data regarding the low-temperature specific heat. A standard closed-cycle refrigerator was employed for measurements using the MARI spectrometer. In such set of experiments the hydrogenated samples were held within a thin aluminum plate, whereas a standard annular corona was used for the deuterated samples.

A rough evaluation of the multiple-scattering effects was carried by means of runs using the DISCUS code ${ }^{28}$ employing the spectrum as calculated by lattice dynamics as a scattering kernel. Multiply scattered neutrons were found to contribute, for the hydrogenous samples, less than six percent. In addition, most of the multiply scattered intensity was found to be located at frequencies about the elastic peak. In consequence, no detailed correction for multiple scattering was deemed neccessary.

Both instruments are direct-geometry chopper spectrometers, with incident energies of $\approx 2 \mathrm{THz}$ (IN5) and $20 \mathrm{THz}$ (MARI) chosen to excite the relevant part of the vibrational spectrum at low temperatures.

The derivation of the $Z(\omega)$ frequency distributions from the measured neutron intensities involved a number of steps such as (a) an angular average over the spectra obtained for different angles, (b) the subtraction of the contribution from the empty container, (c) the removal of the residual elastic contamination at low frequencies (below $50 \mathrm{GHz}$ ) and extrapolation to zero frequency by a $\omega^{2}$ law, (d) the estimation of a multiphonon excitation following the procedure described in Ref. 29 and subtraction of this contribution to the total spectrum. This is required since such effects become noticeable even at low temperatures due to the relatively large frequencies involved. As a final step (e), the normalization to an absolute scale. To achieve this, the spectra measured up to large energy transfers were first normalized to the number of degrees of freedom that were deemed relevant on the basis of the lattice dynamics calculation. A final normalization was made by comparison with the measured specificheat curves, as explained below.

\section{Lattice dynamics}

Ethanol shows a monoclinic $P c$ stable crystal phase with cell parameters $a=5.377 \AA, b=6.882 \AA, c=8.255 \AA$, and $\beta=102.2^{\circ}$ at $87 \mathrm{~K}$ with four molecules per unit cell. ${ }^{30}$ There are two independent molecular configurations in the unit cell not related by symmetry, where half the molecules show a gauche geometry (conformation) with a $\mathrm{C}-\mathrm{C}-\mathrm{O}-\mathrm{H}$ torsion (dihedral) angle of $-63(2)^{\circ}$ and the other half shows a trans conformation with a torsion angle of $179(2)^{\circ}$. The conformation about the $\mathrm{C}-\mathrm{C}$ bond is staggered in both molecules and none of the $\mathrm{O}-\mathrm{C}-\mathrm{C}-\mathrm{H}$ torsion angles deviates more than
$4 \sigma$ from the perfectly staggered conformation. It is known from microwave experiments that both conformers are present in the gas phase, ${ }^{31,32}$ although ab initio calculations ${ }^{33}$ show that the trans configuration is that stablest in the gas phase. The molecular bond lengths and bond angles of both conformations are similar and the most significant difference occurs in the $\mathrm{C}-\mathrm{C}-\mathrm{O}$ angle, which is $3.1^{\circ}$ larger in the gauche compared to the trans form. The crystal packing shows hydrogen bonding $\mathrm{O}-\mathrm{H} \cdots \mathrm{O}$ where the molecules are linked forming infinite chains.

There are different potential functions that have been developed to be used in simulations of liquid alcohols. These proved to be inadequate for modeling the crystal structure since they gave an energy-minimized crystal configuration that departed significantly from experiment. In particular, a good number of them ${ }^{34}$ collapses the $\mathrm{CH}_{3}$ group into a single force center, whereas an adequate representation of a molecular crystal, both in terms of the structure and the lattice dynamics, requires that the $\mathrm{H}$ atoms be considered as force centers because they are crucial in determining the crystal packing. The potential here used was chosen among those showing a better performance in predicting crystal structures and lattice frequencies. The well-known 6-exp atom-atom potential set of Williams ${ }^{35}$ has been chosen for the intermolecular interactions involving atoms of $\mathrm{C}$ and $\mathrm{H}$ whereas for the $\mathrm{O}$ interactions the parameters of Ref. 36 have been taken. The $\mathrm{O}-\mathrm{H} \cdots \mathrm{O}$ hydrogen-bond interaction deserves special consideration. It has been modeled by means of electrostatic interactions involving the $\mathrm{C}-\mathrm{O}-\mathrm{H}$ groups. Point charges of $-0.285 e, 0.685 e$, and $-0.400 e$ have been ascribed ${ }^{37}$ to $\mathrm{C}$, $\mathrm{O}$, and $\mathrm{H}$ atoms belonging to these groups, respectively, whereas the 6-exp short-range interactions have been omitted in the calculation of the crystal energy for the HJ6O contacts involved in the hydrogen bonds.

We have considered the molecule as semirigid, allowing for certain intramolecular degrees of freedom, those involving skeletal distortions that are liable to produce the lowest molecular frequencies to be mixed with the lattice vibrations. In our case, we have allowed for two internal degrees of freedom, namely, the torsion angle $\phi$ around the $\mathrm{C}-\mathrm{C}$ bond and the $\mathrm{C}-\mathrm{C}-\mathrm{O}$ bond angle $\theta$. For the former, an energy contribution of the form

$$
V(\phi)=\frac{1}{2} K_{\phi}(1+\cos 3 \phi)
$$

has been adopted, whereas for the latter, a harmonic potential

$$
V(\theta)=\frac{1}{2} K_{\theta}\left(\theta-\theta_{0}\right)^{2}
$$

has been chosen. Nonbonded 6-exp intramolecular interactions have also been considered except for 1-3 interactions, i.e., interactions between atoms bonded to a common third one. The torsional energy constant $K_{\phi}$ has been fitted in order to reproduce the crystal conformation in an energy minimization process, whereas for the $K_{\theta}$ constant both the crystal structure and Raman frequencies of the bending modes have been used in the adjustment. The resulting values for these constants are $K_{\phi}=7.62 \mathrm{~kJ} \mathrm{~mol}^{-1}$ and $K_{\theta}$ $=0.155 \mathrm{~kJ} \mathrm{~mol}^{-1}$, which are close to others found for glycerol. $^{38}$

A crystal energy minimization gave results within the acceptable tolerance limits. The lattice parameters $a, b$, and $c$ 


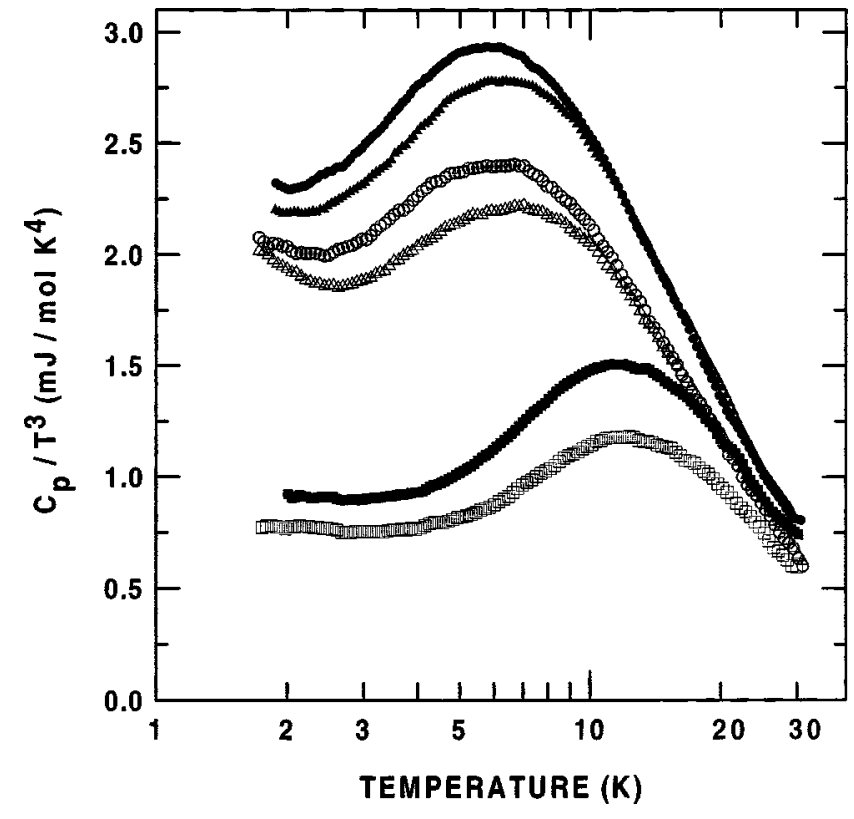

FIG. 3. Low-temperature specific heat of stable crystal ( $\square)$, orientational glass $(\triangle)$, and true glass $(\bigcirc)$, for normal ethanol $\mathrm{CH}_{3} \mathrm{CH}_{2} \mathrm{OH}$ (open symbols) and fully-deuterated ethanol $\mathrm{CD}_{3} \mathrm{CD}_{2} \mathrm{OD}$ (full symbols).

changed in $4.5,5.9$, and $2.3 \%$ respectively, and the calculated $\beta$ angle is $101^{\circ}$. The maximum molecular rotational displacement was $4.8^{\circ}$ and the maximum translational component was $0.1 \AA$. The torsion C-C angles of both independent molecules varied by $3.2^{\circ}$ and $0.6^{\circ}$ and the changes of the bending angle $\mathrm{C}-\mathrm{C}-\mathrm{O}$ were $3.0^{\circ}$ and $2.7^{\circ}$.

\section{RESULTS}

\section{A. Calorimetry}

The results of the specific-heat measurements between 1.8 and $30 \mathrm{~K}$ are shown in Fig. 3. Both structural and orientational-glass solids are there shown to exhibit ${ }^{23,24}$ the anomalous glassy features: a minimum in $C_{p} / T^{3}$ at about 2 $\mathrm{K}$, separating a rise at lower temperatures usually ascribed to tunneling states in glasses, and another, more debated, increase at higher temperatures leading to the maximum in $C_{p} / T^{3}$. On the contrary, the monoclinic crystals show a typical Debye behavior arising from long-wavelength acoustic phonons: $C_{p} \propto T^{3}$ at the lowest temperatures, with a gradual deviation at higher temperatures arising from the increase in VDOS of the lower transverse-acoustic branches near the Brillouin-zone boundaries, which occurs at about $1.5 \mathrm{THz}^{24}$ In general, the deuterated phases of ethanol are somewhat softer than the corresponding hydrogenous counterparts, exhibiting an enhanced specific heat at low temperature, which is explainable by the mass difference and with their maxima in $C_{p} / T^{3}$ shifted to lower temperatures.

In Fig. 4, the low-temperature specific heat of stable crystalline phases of hydrogenated and fully-deuterated ethanol are shown, together with the curves calculated from the VDOS derived from neutron scattering, as well as calculations from lattice dynamics. The Debye temperatures $\Theta_{D}$ are obtainable from the cubic term of the specific heat. We find $\Theta_{D}=284 \pm 3 \mathrm{~K}$ for $\mathrm{CH}_{3} \mathrm{CH}_{2} \mathrm{OH}$ and $\Theta_{D}=268 \pm 2 \mathrm{~K}$ for

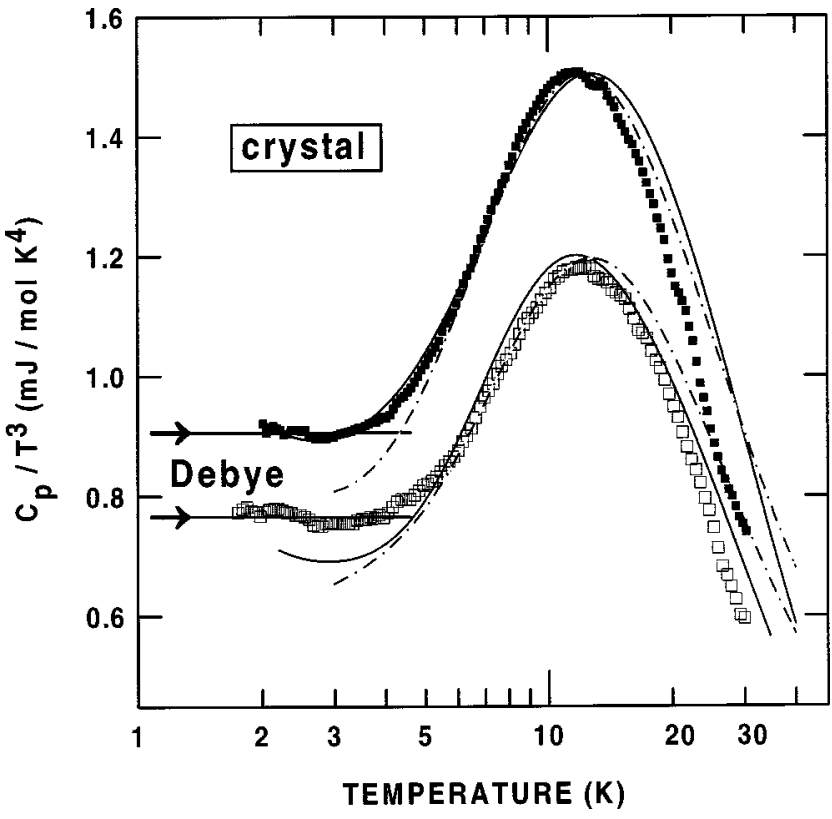

FIG. 4. Low-temperature specific heat of the stable monoclinic crystals for normal ethanol $\mathrm{CH}_{3} \mathrm{CH}_{2} \mathrm{OH}$ (open symbols) and fullydeuterated ethanol $\mathrm{CD}_{3} \mathrm{CD}_{2} \mathrm{OD}$ (full symbols). The fitted $T^{3}$ Debye contributions at the lowest temperatures are also indicated. Solid lines correspond to calculations of the specific heat derived from the neutron-scattering VDOS data. Dotted-dashed lines correspond to LD calculations, multiplied by a scaling 1.35 factor (see text for details).

$\mathrm{CD}_{3} \mathrm{CD}_{2} \mathrm{OD}$. That is, the low-temperature specific heat of deuterated ethanol $C_{\text {Debye }}^{D}$ is $18 \%$ larger than that of hydrogenated ethanol $C_{\text {Debye }}^{H}$, i.e., $C_{\text {Debye }}^{D} / C_{\text {Debye }}^{H}=1.18$. The molecular weights of normal and fully-deuterated ethanol are $M=46.07 \mathrm{~g} / \mathrm{mol}$ and $M=52.12 \mathrm{~g} / \mathrm{mol}$, respectively. Therefore, it can be found that $C_{\text {Debye }} \propto M^{3 / 2}$ and so $\Theta_{D} \propto M^{-1 / 2}$, approximately. Since the sound velocities are proportional to the inverse root of mass density, and $\Theta_{D}$ is in turn proportional to the average sound velocity, the isotopic effect in the crystal phase seems to be accountable by the mass difference, provided that the elastic moduli are the same.

As seen in Fig. 4, the position of the maxima and relative heights in $C_{p} / T^{3}$, and therefore the isotopic effects, are well accounted for by the calculations performed using the neutron VDOS on a (quasi-)harmonic basis. Moreover, the experiment is also well accounted for by the calculation employing the frequency distributions computed from the lattice dynamics, provided that a global scale factor of about 1.35 is used. This comes as a consequence of some additional degrees of freedom that have small contributions to the specific heat, which are not explicitly accounted for in the calculation, as will be commented on below.

Figure 5 shows the results of our measurements for both hydrogenous and deuterated structural glasses. Specific-heat curves calculated from the VDOS given by our lowtemperature neutron-scattering data are also shown in Fig. 5. The same kind of representation is displayed in Fig. 6 for the orientational glasses. Data shown in both figures are strikingly similar. Both conventional glasses (frozen-in supercooled liquids) and orientational glasses (rotationally frozen plastic crystals) exhibit typical glassy features in the specific 


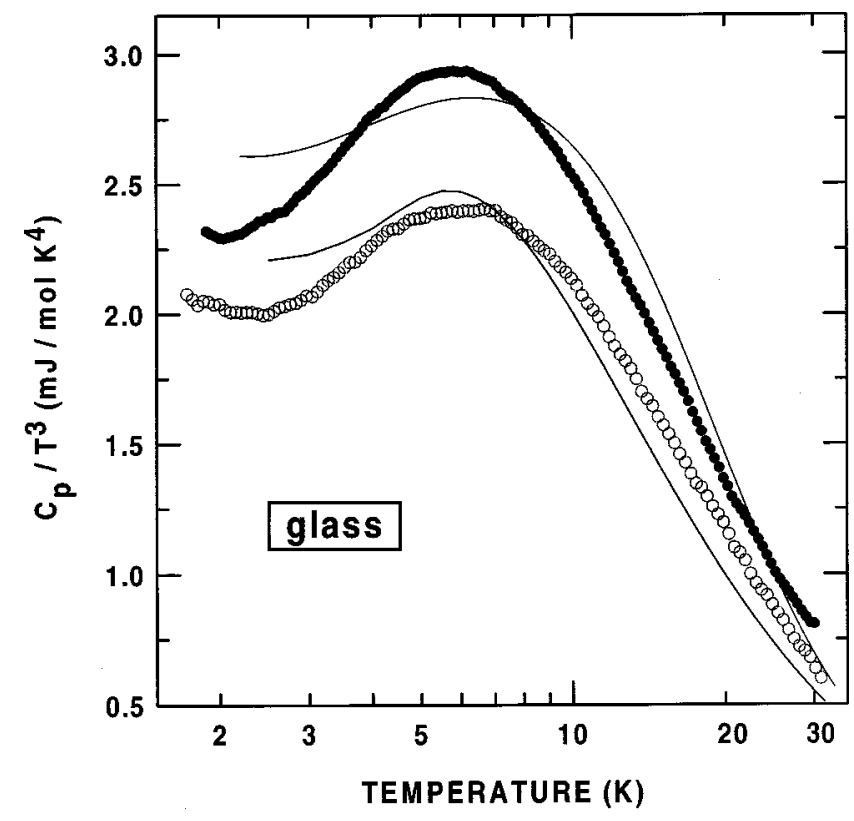

FIG. 5. Low-temperature specific heat of structural glasses prepared from normal ethanol $\mathrm{CH}_{3} \mathrm{CH}_{2} \mathrm{OH}$ (open symbols) and from fully-deuterated ethanol $\mathrm{CD}_{3} \mathrm{CD}_{2} \mathrm{OD}$ (full symbols). Solid lines correspond to calculations of the specific heat derived from the neutron-scattering VDOS data.

heat, even showing remarkably close isotopic effects. Some minor differences between the two glassy solids are to be noticed: the true structural glasses have an additional $10 \%$ excess of specific heat, and their features are shifted to lower temperatures compared to orientational glasses, which, lacking positional disorder, have fewer configurational degrees of freedom, as demonstrated by comparing their specific

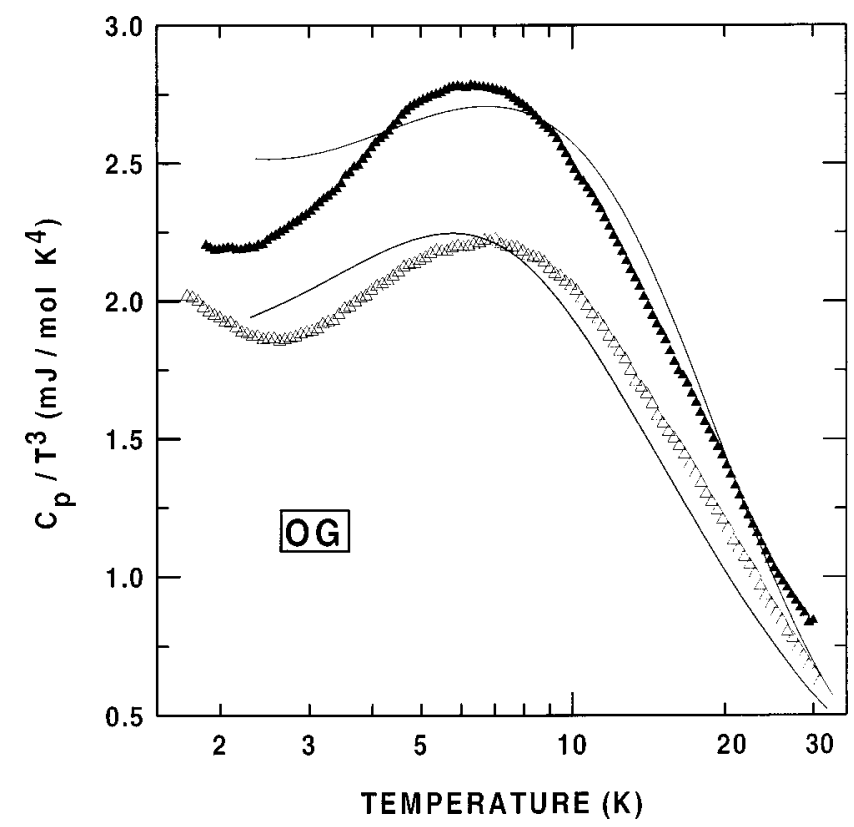

FIG. 6. Low-temperature specific heat of orientational glasses (OG phases) prepared from normal ethanol $\mathrm{CH}_{3} \mathrm{CH}_{2} \mathrm{OH}$ (open symbols) and from fully-deuterated ethanol $\mathrm{CD}_{3} \mathrm{CD}_{2} \mathrm{OD}$ (full symbols). Solid lines correspond to calculations of the specific heat derived from the neutron-scattering VDOS data.

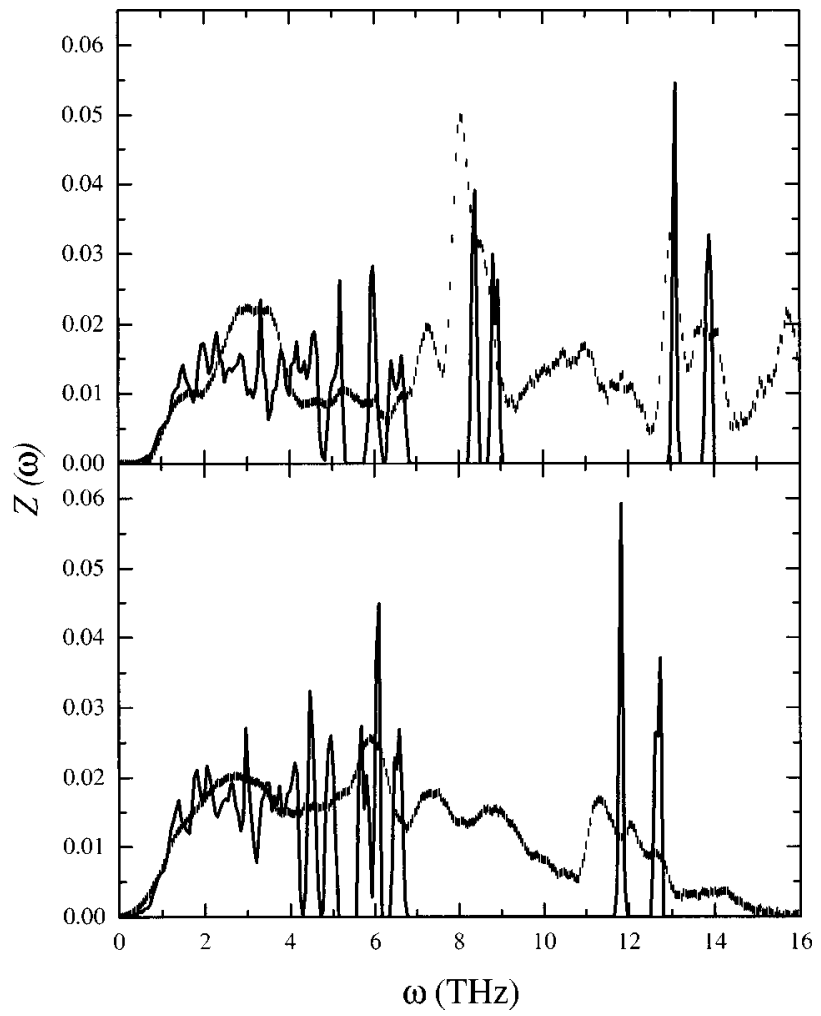

FIG. 7. Comparison between the frequency distributions measured for the stable crystal phase (symbols) and those calculated by means of harmonic lattice dynamics (solid) procedures described in the text. The upper frame depicts data for the hydrogenous samples, whereas the lower frame shows the distributions for fullydeuterated ethanol.

jumps at the glass transitions (see Fig. 2). As evidenced by the comparison between calorimetric data and those calculated from the frequency distributions, the measured VDOS contain all the relevant dynamic information needed to account for the specific heat above a few kelvins.

As referred to above, the "glass transition" observed in these solids is not an ordinary, canonical glass transition, but rather a $\mathrm{RP} \rightarrow \mathrm{OG}$ dynamical transition leading to a freezing of the molecular rotational motions. The striking qualitative and quantitative similarities between both transitions suggest ${ }^{21,22,24,26}$ some important consequences for our understanding of the glass transition. In fact, the present data show that most of the configurational degrees of freedom that show an abrupt change at the canonical glass transition are identifiable with those associated with dynamical freezing into orientational disorder. When positional disorder characteristic of amorphous solids is further introduced, it is seen to amount to a mere, about $20 \%$, change in the specific-heat jump.

The same qualification, that is, the prominent role of molecular orientational correlations in driving the glassy dynamics, applies to the VDOS derived from neutron scattering, as will be shown in the following paragraphs.

\section{B. Frequency distributions}

The calculated and measured frequency distributions for the monoclinic crystals are shown in Fig. 7. As expected, the agreement between calculation and experiment has to be 


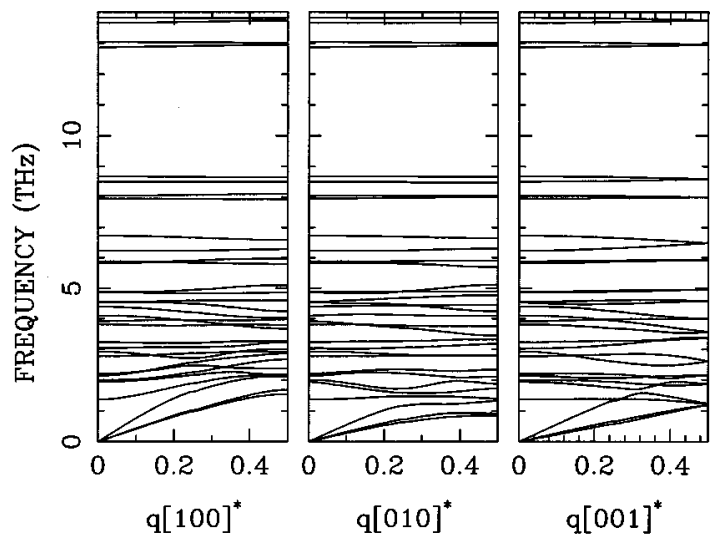

FIG. 8. Calculated dispersion relations for the three highersymmetry directions of the monoclinic crystal.

considered as semiquantitative. The region below $9 \mathrm{THz}$ in the hydrogenous compound $(\mathrm{H})$ and that below $7 \mathrm{THz}$ in the fully deuterated (D) stand a more favorable comparison than the full spectra, mainly because the complicated shapes appearing at frequencies $9-12.5 \mathrm{THz}$ in the hydrogenated sample and $7-11 \mathrm{THz}$ in the deuterated crystal. These mostly arise from rotations of the $\mathrm{OH}$ group (expected at about 9.1 and $8.5 \mathrm{THz}$ for both trans and gauche conformers in the hydrogenous gas-phase compound ${ }^{33}$ ), which are not accounted for by the LD model, as well as from combination bands of some of the fundamentals, which are not considered in a purely harmonic calculation. Between the latter, the strong peaks appearing in the calculation and experiment, about $8 \mathrm{THz}(\mathrm{H})$ and $6 \mathrm{THz}(\mathrm{D})$ arise from the internal torsional librations about the $\mathrm{C}-\mathrm{C}$ bond angle. This can be asserted on the basis of the LD calculation, the intensity ratio of the peaks upon deuteration as well as from the isotopic effect. Their relative frequencies are not too far from those derived from quantum-chemical calculations ${ }^{33}$ that predict free-molecule values of $7.3 \mathrm{THz}(\mathrm{H})$ and $5.5 \mathrm{THz}(\mathrm{D})$, respectively. This, together with the complicated shape appearing within the referred frequency region that shows at least three extrema, serves to illustrate the nontrivial results of the interaction of this coordinate with the lattice (external) modes. The multiplet structure at about $13-14 \mathrm{THz}(\mathrm{H})$ and $12-13 \mathrm{THz}$ (D) corresponds to the higher molecular frequency, which involves C-C-O skeletal deformations. These compare with the free-molecule values of 12.3 and $12.5 \mathrm{THz}$ for both conformers in the $(\mathrm{H})$ form and 10.7 and $10.9 \mathrm{THz}$ in the deuterated sample.

Because of the complicated molecular dynamics, even if a semirigid model for the molecule is used, the assignment of extrema of $Z(\omega)$ to modes with a well-defined character (i.e., polarization) becomes an unattainable task. However, the results from the LD calculations allow setting some dominant character for most peaks as well as serve to establish different frequency ranges as characteristic of the various kinds of atomic motions. With such an aim, Fig. 8 shows the calculated 32 dispersion branches along the crystal highersymmetry directions. As easily seen there, the acoustic branches are confined to a region below $2 \mathrm{THz}$, above which lies a dense mesh of mildly dispersive modes. A correlation between some of the features in the $Z(\omega)$ and the calculated dispersion curves reveals that (a) the lowest-frequency extre- mum corresponding to acoustic modes is seen as a shoulder at about $1 \mathrm{THz}$ in Fig. 7 and corresponds to zone-boundary TA phonons in the [010] and [001] directions, (b) the first well-defined peak that appears at about $1.5 \mathrm{THz}$ in the VDOS no longer can be associated with purely acoustic modes since both longitudinal and transverse acoustic and the lower-lying optic branches contribute significantly to this peak, (c) all peaks above these appearing at $2.1 \mathrm{THz}$ are to be considered as purely optic in character.

On the other hand, the mode eigenvectors of the 29 nontrivial modes arising from the LD calculation also provide additional information on the atomic dynamics. A table of $\mathrm{Q} \rightarrow 0$ has been generated ${ }^{39}$ containing the eigenvector components of each mode in terms of six rigid-body molecular translations and rotations and the two bond-torsion and bond-bending internal coordinates explicitly considered in the calculation. Examination of the mode-normalized eigenvector coefficients soon reveals that (a) the normal modes start to show an important deformational component, as defined by eigenvector components higher than 0.1 at frequencies as low as $1.8 \mathrm{THz}(\mathrm{D})$ that is about three times lower than the free-molecule value, (b) most of the modes between such frequency and $6.6 \mathrm{THz}$ carry a substantial torsional component, and (c) even the bond-bending mode shows noticeable hybridization with the lattice modes that leads to the appearance of four modes with frequencies between 11.6 and $12.6 \mathrm{THz}$, instead of only two expected on the basis of freemolecule frequencies for both trans and gauche conformers.

The frequency distributions for the orientational-glass solids in both $\mathrm{H}$ and $\mathrm{D}$ forms are shown in Fig. 9. An interesting thing to notice is the relatively small effect that the loss of orientational order has on the internal molecular vibrations which, apart from a very substantial broadening, leaves the two bands centered at frequencies close to those of the sharp monoclinic-crystal peaks. This indicates that the main effect on the internal molecular low-frequency dynamics introduced by the loss of orientational order concerns the $K_{\phi}$ and $K_{\theta}$ force constants that are now distributed over a range of values about those that serve to describe the monoclinic crystal. In consequence, it is expected that all qualifications discussed in the previous section regarding the hybridization between lattice and internal motions will also apply to phases devoid of long-range order.

In stark contrast with this, the region of lower frequencies becomes markedly different from that of the stable crystals, as attested by the increase in intensity below $1 \mathrm{THz}$ over the $\omega^{2}$ law shown by the fully-ordered crystal and the loss of the shoulders that appeared at about $1.5 \mathrm{THz}(\mathrm{H})$ and $1.2 \mathrm{THz}$ (D). As shown by comparisons of the low-temperature specific heats given above, these regions of low frequencies are precisely those that contain the dynamical information responsible for the specific-heat anomalies.

A comparison of the rotator crystal spectrum, shown in the lower frame of Fig. 9, with those shown above for the rotationally-frozen phases, serves to illustrate how the welldefined finite-frequency features of the latter are lost because of both the emergence of molecular rotations and the hightemperature, which leads to substantial broadenings of most peaks, as seen even for the stable crystal.

Finally, the frequency distributions for the fully disordered glass are shown in Fig. 10. A comparison between the 


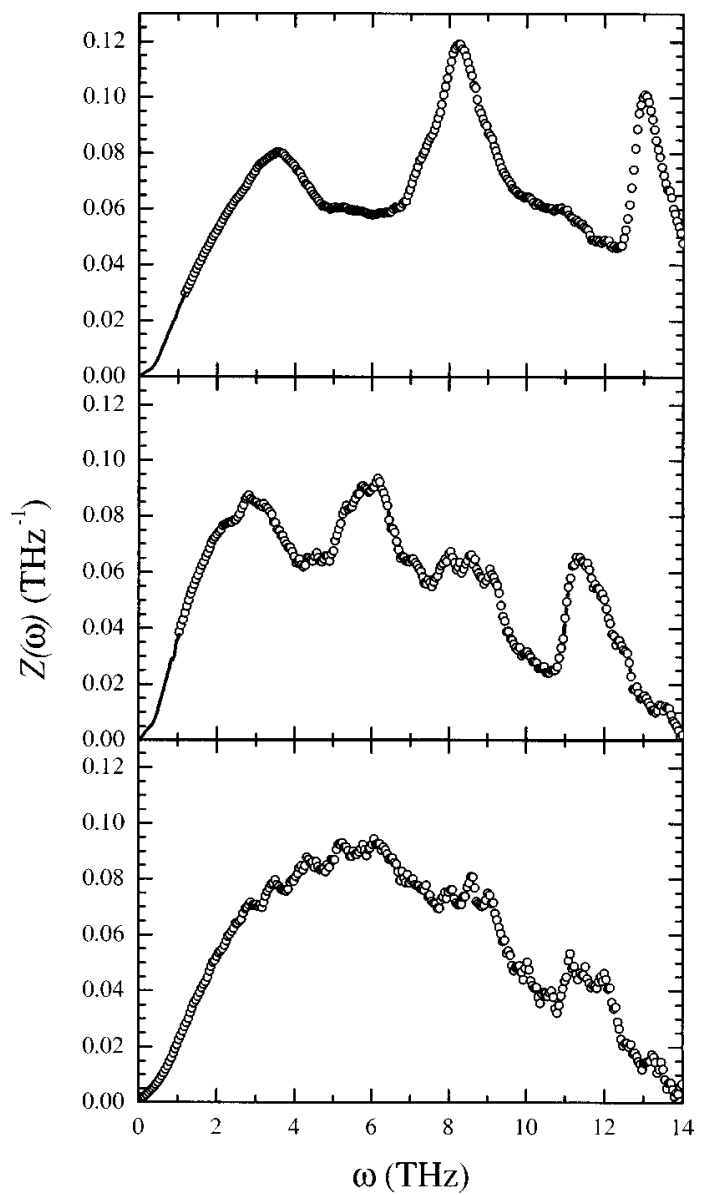

FIG. 9. Vibrational densities of states for the orientational-glass phases. The upper frame shows the VDOS for fully-hydrogenated sample at $5 \mathrm{~K}$ and that in the middle frame depicts data for the fully-deuterated sample. Symbols represent data measured using the MARI spectrometer and the line at low-frequencies those measured at IN5. The lower frame displays the spectrum of the rotator-phase crystal measured at $107 \mathrm{~K}$.

distributions of the glass and OG crystals has already been reported $^{24}$ and shows a striking similitude, a fact also exemplified by the specific heat. Therefore, the main purpose of the data shown in the figure referred to is to illustrate the extent up to which the isotopic effect can be followed. A comparison of the low-frequency tails of the distributions there shown reveals that such a difference can be followed down to $0.12 \mathrm{THz}$. This compares with data for the monoclinic and OG crystals that show that isotopic differences can be traced down to 0.4 and $0.15 \mathrm{THz}$ respectively. The frequency distributions depicted in Fig. 10 show a complicated $\omega$ dependence which, on empirical grounds and below about $0.25 \mathrm{THz}$, is well accounted for by a linear and a quadratic term, the latter having a coefficient 3-4 times larger than the former. Inspection of the graphs referred to also reveals that the deuterated samples have an excess intensity over the hydrogenous at frequencies between $0.12 \mathrm{THz}$ and $\approx 0.8 \mathrm{THz}$, where the distributions cross each other. Such isotopic effects seem far larger than that seen for the monoclinic crystals, and therefore cannot be fully ascribed to a simple molecular-mass effect. To assess the physical relevance of such differences, their effect on the specific heats calculated on the harmonic approximation was examined. In doing so,

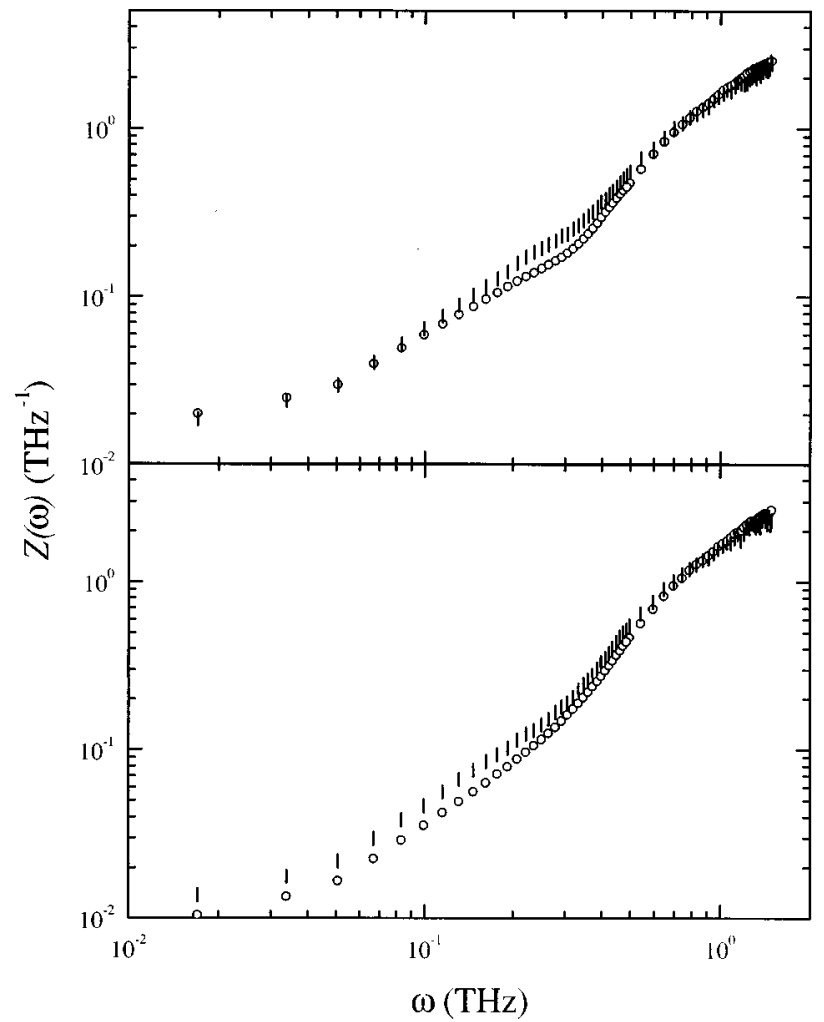

FIG. 10. Vibrational densities of states for the structural glasses. The upper frame shows data at $5 \mathrm{~K}$ on IN5 and the lower frame those measured at $20 \mathrm{~K}$. In both frames the circles represent data for the deuterated compound and vertical bars depict the fully hydrogenous sample.

ratios of 1.3-1.4 times the specific heat of the deuterated and hydrogenous compounds were found. These are far larger than those encountered for the monoclinic crystal, which are of 1.08 and can be accounted for by the difference in molecular masses. In fact, the only other spectroscopic quantity showing such a large isotopic effect concerns the molecular low-energy torsional motion that shows an isotopic shift of 1.33 in the trans and 1.40 for the gauche species.

\section{DISCUSSION}

The data and arguments given in the present work should serve to establish a quantitative comparison between the dynamic anomalies shown by two glassy forms of the same molecular compound. In particular, evidence here is provided showing that the periodicity that once lost, drives the dynamics of the solid into the "glassy" realm, mostly concerns the relative molecular orientations. A glance to the molecular structure of the monoclinic crystal shows the molecular units arranged in parallel, zig-zag chains with trans and gauche conformers as alternate links. Such long-range orientational ordering corresponds to a crystal some 1.15 times more dense than the glass or the OG solids that is strongly stabilized by orientational interactions of electricdipole origin. In fact, full orientational order is achieved as a consequence of attractive electrostatic interactions between dipoles of $1.7 \times 10^{-18}$ esu $\mathrm{cm}$ and about $1.8 \times 10^{-18}$ esu cm located in the trans and gauche conformers. Such interactions can, under annealing conditions, overcome the entropy- 
driven processes that marginally stabilize the supercooled liquid, and lead to the nucleation of crystallites with monoclinic structure.

A number of calculations carried out for several configurations of an ethanol trans-gauche dimer ${ }^{33}$ have unveiled a rather complex potential-energy surface showing at least two minima in addition to that of the monoclinic crystal. A configuration resembling the latter was found to be about $4 \mathrm{THz}$ above the minimum. The other two, which differ in energy by $0.4 \mathrm{THz}$, may be accommodated within a bcc lattice such as that shown by the OG. The multiple-minima surface happens as a consequence of competing electrostatic and van der Waals interactions, the former forcing the electric dipoles to align whereas the latter shows a prominent dependence of the molecular shapes. Within the condensed phases the latter can be identified with that forcing the optimization of the excluded volume (i.e., the gauche form shows a significantly smaller molecular volume than the trans). The most glaring evidence of such an interplay of forces is given by the different ratios of gauche/trans particles appearing in the different phases ${ }^{22}$ that departs from the unit ratio characteristic of the monoclinic crystal.

The internal low-frequency torsional motion thus seems to play a role as a source of frustration that increases the glass-forming ability of the material. In fact, a chemically close material such as methanol $\left(\mathrm{CH}_{3} \mathrm{OH}\right)$, devoid of such internal flexibility, cannot be easily glassified from the supercooled liquid state unless small amounts of water are present. ${ }^{40}$ In analogy with the present case, the water impurity that has an electric dipole moment of $1.84 \times 10^{-18}$ esu $\mathrm{cm}$, not too different from the $1.70 \times 10^{-18} \mathrm{esu} \mathrm{cm}$ of methanol, also shows a substantially different molecular volume.

The low-energy excitations typical of glasses are ascribed to the excess over the Debye background, which can be reasonably related to the minimum value, since the exact value is not known. The difference between the maximum and the minimum in $C_{p} / T^{3}$ seems therefore a sensible measure of the amount of the anomalous excess contribution to the $C_{p}$ characteristic of glasses. As seen in Figs. 5 and 6, there exist only minor differences between glasses with or without positional disorder: the true structural glasses have about $10 \%$ larger excess of specific heat, and their features are shifted to lower temperatures compared to orientational glasses that lack positional disorder and therefore possess a few less configurational degrees of freedom, as demonstrated by comparing their specific-heat jumps at the glass transitions (see Fig. 2).

As regards the remarkable isotopic effects shown in the VDOS of the glassy solids, it seems worth noticing that such findings are also borne out by the calorimetric measurements. Interestingly, the specific-heat values at the minimum in $C_{p} / T^{3}$ give isotopic ratios close to those found for the stable crystal with 1.15 for glass and 1.18 for the OG. Since the specific heat at this point is basically the Debye contribution (plus the smallest possible excess contribution), it indicates that $C_{\text {Debye }} \propto M^{3 / 2}$ also applies for all the glassy states. This contrasts with what is found for the bump in $C_{p} / T^{3}$, especially after subtraction of the Debye contributions. The relative heights of these are about as large as 1.6 for glass and 1.7 for the OG, whereas a ratio of only 1.08 is found from the maxima for the stable crystals, accountable in terms

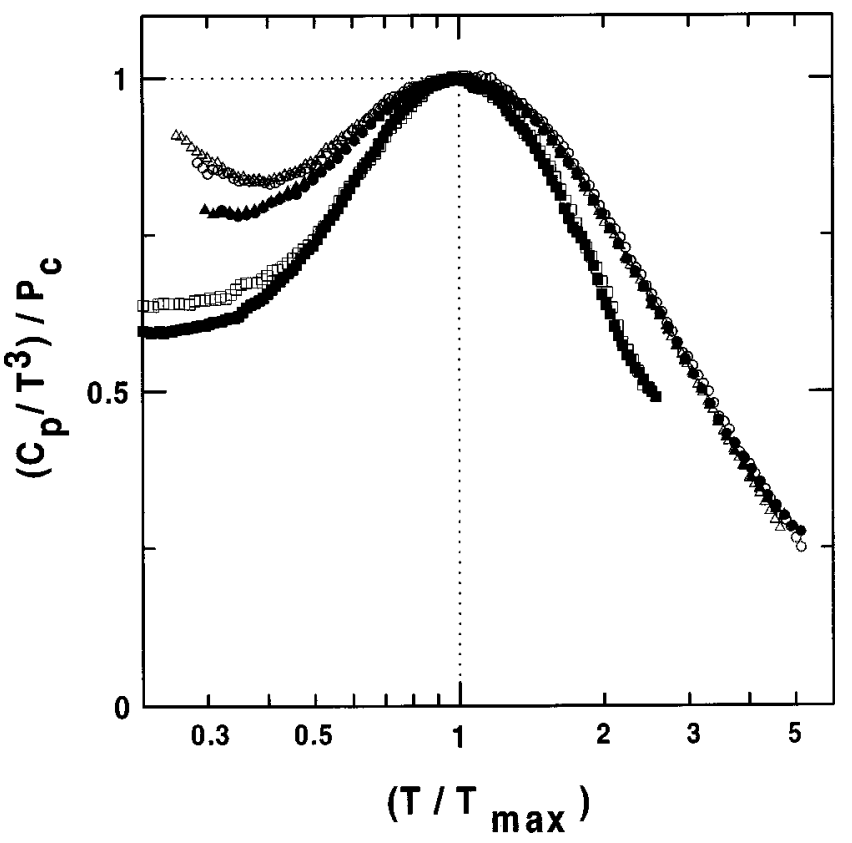

FIG. 11. Scaling of the specific-heat curves to their maxima, following Liu and von Löhneysen (Ref. 41), as $\left(C_{p} / T^{3}\right) / P_{c}$ vs $T / T_{\max }$. The symbols are the same used in previous figures: stable crystal $(\square)$, orientational glass $(\triangle)$, and true glass $(\bigcirc)$, for normal ethanol $\mathrm{CH}_{3} \mathrm{CH}_{2} \mathrm{OH}$ (open symbols) and fully-deuterated ethanol $\mathrm{CD}_{3} \mathrm{CD}_{2} \mathrm{OD}$ (full symbols).

of concomitant changes in the molecular moments of inertia.

Finally, Fig. 11 shows a graph of $\left(C_{p} / T^{3}\right) / P_{c}$ versus $T / T_{\max }$, where $P_{c}$ is the height of the maximum in $C_{p} / T^{3}$ and $T_{\max }$ its position. The scaling follows that of Liu and von Löhneysen ${ }^{41}$ employed for several glasses and shows that data for the disordered solids superimpose in the whole temperature region. In contrast, the curves for the ordered crystals show a far narrower shape, in agreement with similar findings in other materials. ${ }^{42}$ This fact again evidences the different nature of the low-energy vibrational spectra of glasses and ordered crystals. Very interestingly, the behavior of deuterated glasses (either structural or orientational) deviate from that of the hydrogenous ones at low temperatures, in quite the same way as the deuterated monoclinic crystal does from the hydrogenous one.

\section{SUMMARY AND CONCLUSIONS}

The results given in this work provide a quantitative confirmation of the fact that glassy behavior is not an exclusive property of amorphous solids, which simply lack translational disorder, but rather of materials usually referred to as soft matter. Also, the limited amount of low-temperature data available serves to provide an answer to the question of whether the low-temperature anomaly [linear contribution to $\left.C_{p}(T)\right]$ was an intrinsic property of the amorphous state. Such a question, posed more than a decade ago (see Pohl in Ref. 3, p. 36), does not seem to have yet been answered quantitatively. As proven by both calorimetric and spectroscopic means, an orientationally-disordered crystal shows "glassy" features to an extent remarkably close to that shown by the amorphous solid, at both low and intermediate temperatures. 
A common characteristic of materials showing a welldefined calorimetric glass transition (i.e., that excludes most metallic glasses), is the presence of configurational degrees of freedom that freeze-in at the glass-transition temperature additional to those corresponding to rigid-body motions of their constituent particles. In this respect, a further identification of such degrees of freedom with those that at low temperatures are describable as the soft modes responsible of the universal anomalous thermal and vibrational behavior of glasses, seems justified here. By implication, it should then be expected that some special amorphous solids such as pure $a-\mathrm{Si}$, with very little flexibility, may not exhibit a pronounced glassy behavior, whereas the converse applies to many other orientational glass crystals.

The results described in this work should then be of relevance for a wide class of glass-forming materials irrespective of having well-defined molecular entities as their constituent particles. Such an assertion is supported by recent work on some other glasses of covalent origin such as vitre- ous silica. ${ }^{43}$ There, the presence of low-frequency motions involving the concerted motions of relatively large groups of atoms, termed "rigid unit modes," seems to be the source of the dynamical anomalies observed in some disordered forms of silica, such as the presence of a boson peak in a disordered crystal state ( $\alpha$ cristobalite), as well as a large excess in specific heat at intermediate temperatures. ${ }^{44}$ The data presented here go a step further in quantifying the effects of disorder on glassy dynamics since, contrary to the case of $\alpha$ cristobalite, a low-temperature anomaly is prominent in the disordered crystal states. In addition, both the disordered crystal, glass, and fully-ordered solids are studied under the very same thermodynamic conditions.

\section{ACKNOWLEDGMENTS}

This work was supported in part by DGICYT (Spain) Grant No. PB95-0075-c03-01 and by the EU within INTAS Project No. 93-3230.
${ }^{1}$ R. C. Zeller and R. O. Pohl, Phys. Rev. B 4, 2029 (1971).

${ }^{2}$ R. B. Stephens, Phys. Rev. B 8, 2896 (1973); 13, 852 (1976).

${ }^{3}$ Amorphous Solids: Low Temperature Properties, edited by W. A. Phillips (Springer, Berlin, 1981); W. A. Phillips, Rep. Prog. Phys. 50, 1657 (1987).

${ }^{4}$ K. A. Topp and D. G. Cahill, Z. Phys. B 101, 235 (1996). Also, B. E. White and R. O. Pohl, ibid. 100, 401 (1996).

${ }^{5}$ D. Richter, J. Phys.: Condens. Matter 8, 9177 (1996), and references therein.

${ }^{6}$ W. A. Phillips, J. Low Temp. Phys. 7, 351 (1972); P. W. Anderson, B. I. Halperin, and C. M. Varma, Philos. Mag. 25, 1 (1972).

${ }^{7}$ Some recent results extend the range of validity of the tunneling model up to temperatures of 20-40 K, above which the finite occupation of the excited states in the double-well potentials results in thermally activated relaxation. Between 5 and $20 \mathrm{~K}$, the motion of two-level systems can be accounted for by incoherent tunneling. For an account of this and its relationship with the soft-potential model, see A. Würger, From Coherent Tunneling to Relaxation (Springer, Berlin, 1997), p. 115.

${ }^{8}$ D. G. Cahill and R. O. Pohl, Phys. Rev. B 35, 4067 (1987).

${ }^{9}$ See, for instance, papers in Proceedings of the First International Discussion Meeting on Relaxations in Complex Systems [J. NonCryst. Solids 131-133 (1991)]; Proceedings of the Second International Discussion Meeting on Relaxations in Complex Systems [J. Non-Cryst. Solids 172-174 (1994)]; Proceedings of the Third International Discussion Meeting on Relaxations in Complex Systems [J. Non-Cryst. Solids (to be published)]. See also, Disorder Effects on Relaxational Processes, edited by R. Richert and A. Blumen (Springer, Berlin, 1994); Nonequilibrium Phenomena in Supercooled Fluids, Glasses and Amorphous Materials, edited by M. Giordano, D. Leporini, and M. P. Tosi (World Scientific, Singapore, 1996); Complex Behaviour of Glassy Systems, edited by M. Rubí and C. Pérez-Vicente, Springer Lecture Notes in Physics Vol. 492 (Springer, Berlin, 1997).

${ }^{10}$ C. A. Angell, Science 267, 1924 (1995).

${ }^{11}$ A. P. Sokolov, Science 273, 1675 (1996).

${ }^{12}$ L. Gil, M. A. Ramos, A. Bringer, and U. Buchenau, Phys. Rev.
Lett. 70, 182 (1993); M. A. Ramos, L. Gil, A. Bringer, and U. Buchenau, Phys. Status Solidi A 135, 477 (1993).

${ }^{13}$ A. P. Sokolov, E. Rössler, A. Kisliuk, and D. Quitmann, Phys. Rev. Lett. 71, 2062 (1993); A. P. Sokolov, A. Kisliuk, D. Quitmann, and E. Duval, Phys. Rev. B 48, 7692 (1993).

${ }^{14}$ K. Knorr and A. Loidl, Phys. Rev. B 31, 5387 (1985).

${ }^{15}$ J. J. De Yoreo, W. Knaak, M. Meissner, and R. O. Pohl, Phys. Rev. B 34, 8828 (1986).

${ }^{16}$ S. K. Watson, Phys. Rev. Lett. 75, 1965 (1995).

${ }^{17}$ E. R. Grannan, M. Randeria, and J. P. Sethna, Phys. Rev. B 41, 7799 (1990).

${ }^{18}$ The Plastically Crystalline State (Orientationally-Disordered Crystals), edited by J. N. Sherwood (Wiley, New York, 1978).

${ }^{19}$ M. Descamps, M. Bée, P. Derollez, J. F. Willart, and L. Carpentier, in Quasielastic Neutron Scattering, edited by J. Colmenero, A. Alegría, and F. J. Bermejo (World Scientific, Singapore, 1994), pp. 107-127.

${ }^{20}$ O. Haida, H. Suga, and S. Seki, J. Chem. Thermodyn. 9, 1133 (1977).

${ }^{21}$ A. Srinivasan, F.J. Bermejo, A. de Andrés, J. Dawidowski, J. Zúniga, and A. Criado, Phys. Rev. B 53, 8172 (1996).

${ }^{22}$ F. J. Bermejo, A. Criado, R. Fayos, R. Fernández-Perea, H. E. Fischer, E. Suard, A. Guelylah, and J. Zúñiga, Phys. Rev. B 56, 11536 (1997).

${ }^{23}$ M. A. Ramos, Q. W. Zou, S. Vieira, and F. J. Bermejo, Czech. J. Phys. 46, 2235 (1996).

${ }^{24}$ M. A. Ramos, S. Vieira, F. J. Bermejo, J. Dawidowski, H. E. Fischer, H. Schober, M. A. González, C. K. Loong, and D. L. Price, Phys. Rev. Lett. 78, 82 (1997).

${ }^{25}$ It could be mentioned that ethanol exhibits the largest jump in the normalized specific heat at the glass transition reported so far, as can be seen in Fig. 4 of Angell's recent review paper (Ref. 10).

${ }^{26}$ F. J. Bermejo, H. E. Fischer, M. A. Ramos, A. de Andrés, J. Dawidowski, and R. Fayos, in Complex Behaviour of Glassy Systems (Ref. 9), pp. 44-61.

${ }^{27}$ R. Fayos, F. J. Bermejo, J. Dawidowski, H. E. Fischer, and M. A. González, Phys. Rev. Lett. 77, 3823 (1996), and references therein. 
${ }^{28}$ M. W. Johnson, A.E.R.E. Report No. 5697, 1974 (unpublished).

${ }^{29}$ J. Dawidowski, F. J. Bermejo, R. Fayos, R. Fernández-Perea, S. M. Bennington, and A. Criado, Phys. Rev. E 53, 5079 (1996).

${ }^{30}$ P. G. Jönsson, Acta Crystallogr., Sect. B: Struct. Crystallogr. Cryst. Chem. 32, 232 (1976).

${ }^{31}$ J. Michiel-Effinger, J. Mol. Spectrosc. 29, 489 (1969).

${ }^{32}$ Y. Sasada, M. Tanako, and T. Satoh, J. Mol. Spectrosc. 38, 33 (1971).

${ }^{33}$ The estimated differences between trans and gauche forms was found to be strongly dependent upon the particular basis set being employed. The optimal values yielded $1.77 \mathrm{THz}$ for the gauche form with respect to the trans, which compares reasonably well with the experimental estimate of $1.26 \mathrm{THz}$. The binding energy of a trans-gauche dimer was calculated at the 6-31-G $(d, p)$ level. Three different molecular configurations were located in an optimization scheme. A series of calculations of the free-molecule frequencies was carried out by means of quantum-chemical ab initio methods at several levels of sophistication. It was found that large basis sets including polarization and diffuse functions were needed in order to reproduce the experimental, gas-phase frequencies. The quoted values correspond to harmonic frequencies as calculated from the solution of the full molecular vibrational problem employing the basis sets due to D. E. Woon and T. H. Dunning, Jr., J. Chem. Phys. 98, 1358 (1993).

${ }^{34}$ W. L. Jorgensen, J. Phys. Chem. 90, 1276 (1986).

${ }^{35}$ D. E. Williams, J. Chem. Phys. 47, 4680 (1967).

${ }^{36}$ S. R. Cox, D. E. Williams, and L. Hsu, Acta Crystallogr., Sect. A: Cryst. Phys., Diffr., Theor. Gen. Crystallogr. 37, 293 (1981).

${ }^{37}$ W. L. Jorgensen, J. Am. Chem. Soc. 103, 335 (1981).

${ }^{38}$ F. J. Bermejo, A. Criado, A. de Andrés, E. Enciso, and H. Schober, Phys. Rev. B 53, 5259 (1996).

${ }^{39}$ See AIP Document No. E-PAPS: E-PRBMDO-58-056826 for a table of mode-eignevector components. E-PAPS document files may be retrieved free of charge from our FTP server (http:// www.aip.org/epaps/epaps.html) or from ftp.aip.org in the directory /epaps/. For further information: e-mail: PAPS@aip.org or fax: 516-576-2223.

${ }^{40}$ F. J. Bermejo et al., Phys. Rev. B 46, 6173 (1992).

${ }^{41}$ X. Liu and H. von Löhneysen, Europhys. Lett. 33, 617 (1996).

${ }^{42}$ X. Liu and H. von Löhneysen, Phys. Rev. B 48, 13486 (1993).

${ }^{43}$ M. T. Dove, M. J. Harris, A. C. Hannon, J. M. Parker, I. P. Swainson, and M. Gambhir, Phys. Rev. Lett. 78, 1070 (1997).

${ }^{44}$ N. Bilir and W. A. Phillips, Philos. Mag. 32, 113 (1975). 